\title{
Effect of Adhesion and Substrate Elasticity on Neutrophil Extracellular Trap Formation
}

\author{
Luise Erpenbeck ${ }^{1 * x}$, Antonia Luise Gruhn ${ }^{1 \dagger}$, Galina Kudryasheva ${ }^{2}$, Gökhan Günay ${ }^{1,3}$, \\ Daniel Meyer ${ }^{3}$, Julia Busse ${ }^{1}$, Elsa Neubert ${ }^{1,3}$, Michael P. Schön ${ }^{1,4}$, Florian Rehfeldt ${ }^{2}$ and \\ Sebastian Kruss ${ }^{3 *}$
}

${ }^{1}$ Department of Dermatology, Venereology and Allergology, University Medical Center, Göttingen University, Göttingen, Germany, ${ }^{2}$ Third Institute of Physics-Biophysics, Göttingen University, Göttingen, Germany, ${ }^{3}$ Department of Chemistry, Institute of Physical Chemistry, Göttingen University, Göttingen, Germany, ${ }^{4}$ Lower Saxony Institute of Occupational Dermatology, Göttingen, Germany

\section{OPEN ACCESS}

Edited by:

Fabrizio Mattei,

National Institute of Health (ISS), Italy

Reviewed by:

Anderson Guimarães-Costa,

Federal University of Rio de

Janeiro, Brazil

Meraj Alam Khan,

Hospital for Sick Children, Canada

Stavros Giaglis,

University of Basel, Switzerland

*Correspondence:

Luise Erpenbeck

luise.erpenbeck@

med.uni-goettingen.de

Sebastian Kruss

skruss@gwdg.de

†These authors have contributed equally to this work

Specialty section: This article was submitted to

Molecular Innate Immunity,

a section of the journal

Frontiers in Immunology

Received: 20 May 2019

Accepted: 13 September 2019

Published: 01 October 2019

Citation:

Erpenbeck L, Gruhn AL, Kudryasheva G, Günay G, Meyer D, Busse J, Neubert E, Schön MP, Rehfeldt $F$ and Kruss S (2019) Effect of Adhesion and Substrate Elasticity on Neutrophil Extracellular Trap

Formation. Front. Immunol. 10:2320. doi: 10.3389/fimmu.2019.02320
Neutrophils are the most abundant type of white blood cells. Upon stimulation, they are able to decondense and release their chromatin as neutrophil extracellular traps (NETs). This process (NETosis) is part of immune defense mechanisms but also plays an important role in many chronic and inflammatory diseases such as atherosclerosis, rheumatoid arthritis, diabetes, and cancer. For this reason, much effort has been invested into understanding biochemical signaling pathways in NETosis. However, the impact of the mechanical micro-environment and adhesion on NETosis is not well-understood. Here, we studied how adhesion and especially substrate elasticity affect NETosis. We employed polyacrylamide (PAA) gels with distinctly defined elasticities (Young's modulus E) within the physiologically relevant range from 1 to $128 \mathrm{kPa}$ and coated the gels with integrin ligands (collagen I, fibrinogen). Neutrophils were cultured on these substrates and stimulated with potent inducers of NETosis: phorbol 12-myristate 13-acetate (PMA) and lipopolysaccharide (LPS). Interestingly, PMA-induced NETosis was neither affected by substrate elasticity nor by different integrin ligands. In contrast, for LPS stimulation, NETosis rates increased with increasing substrate elasticity $(E>20 \mathrm{kPa})$. LPS-induced NETosis increased with increasing cell contact area, while PMA-induced NETosis did not require adhesion at all. Furthermore, inhibition of phosphatidylinositide 3 kinase (PI3K), which is involved in adhesion signaling, completely abolished LPS-induced NETosis but only slightly decreased PMA-induced NETosis. In summary, we show that LPS-induced NETosis depends on adhesion and substrate elasticity while PMA-induced NETosis is completely independent of adhesion.

Keywords: neutrophil extracellular traps (NET), substrate elasticity, stiffness and its variations, inflammation, immunomodulation, adhesion, innate immunity, neutrophil (PMN)

\section{INTRODUCTION}

Neutrophilic granulocytes are the most abundant type of circulating white blood cells. In a process termed NETosis, they release neutrophil extracellular traps (NETs), web-like structures composed of decondensed chromatin decorated with antimicrobial proteins $(1,2)$. During NETosis, the nuclear chromatin swells until both the nuclear envelope and the outer cell membrane rupture 
(3). NETosis is considered an important immune defense mechanism as neutrophils can bind and kill bacteria and other pathogens via NETs. Apart from physiological stimuli such as pathogens, chemokines (e.g., CXCL8), activated platelets or urea crystals there are several additional NET-inducers like phorbol 12-myristate 13-acetate (PMA) and lipopolysaccharides (LPS), which induce NETosis in vitro (4). Even though NETosis was initially described as part of the innate immune defense system, we know today that dysregulated NETosis is also involved in a variety of chronic inflammatory and autoimmune diseases such as atherosclerosis, systemic lupus erythematosus, preeclampsia, as well as malignant diseases (5-8). Therefore, the question which environmental factors play a role in this process and may influence the course of diseases is highly important.

Mechanical properties of tissues are environmental signals that are able to modulate the functionality of surrounding cells. This has been demonstrated by a substantial amount of studies investigating the effect of physical factors on cellular functions (9-13). It has previously been shown that phenotype and functionality of immune cells such as macrophages and dendritic cells are affected by substrate elasticity/stiffness (14-16). It has also been reported that substrate elasticity affects neutrophil adhesion, migration, and chemotaxis (17-19). Transmigration of neutrophils through endothelium was also proven to be affected by sub-endothelial cell matrix stiffness (20). Tissue stiffness increases in multiple pathological processes including, most prominently, atherosclerotic plaques (21) but also fibrosis (22) and cancer (23). In general, cell adhesion is mediated through surface receptors interacting with specific ligands presented on surfaces (24-26). Integrin ligands have been previously shown to play an important role in leukocyte adhesion and migration (27-29). Additionally, the ligand density on the surface affects adhesion and migration of neutrophils $(28,30)$. For example, neutrophils adhere via the integrin Mac-1 to the platelet receptor GPIb $\alpha$ and show the fastest adhesion maturation when ligands are distributed at a medium distance of approximately 100 nm (28).

In mice, it has been shown that blocking of the integrin LFA1 prevented NETosis in a model of endotoxemia (31). Similarly, mice lacking the beta2 subunit of integrin receptors were largely protected from overwhelming NET production in a murine model of hantavirus infection (32).

In humans, there are several studies that have investigated the involvement of integrin signaling in NET formation, although the results are partially contradictory $(33,34)$. Thus, even though the role of integrins on neutrophil adhesion has been addressed to a certain extent, there is still need to further characterize this complex interaction in a well-defined manner. Moreover, the impact of substrate elasticity in conjuncture with adhesionrelated processes on NETosis has not been investigated yet and thus remains entirely enigmatic.

In this paper, we explore the effect of substrate stiffness/elasticity (Young's modulus $E$ ) and general adhesion on NETosis in human neutrophils (Figure 1). We use collagen Iand fibrinogen-coated polyacrylamide (PAA) gels with stiffnesses within the physiologically relevant range $(E=1-128 \mathrm{kPa})$ to study the impact of elasticity and adhesion on NETosis induced by two different stimuli (LPS, PMA).

\section{MATERIALS AND METHODS}

\section{Polyacrylamide (PAA) Gel Preparation}

Polyacrylamide (PAA) gels were prepared on round glass cover slides as previously reported $(9,13)$. In brief, the cover glasses were plasma cleaned, coated with 3-aminopropyltriethoxysilane (Sigma, Munich, Germany, A3648) and afterwards incubated with glutaraldehyde solution (0.05\%, Sigma, Munich, Germany, G7651). Appropriate mixtures of acrylamide (Bio-Rad, Munich, Germany, \#161-0140) and bis-acrylamide (BioRad, Munich, Germany \#161-0140) diluted in Dulbecco's phosphate-buffered saline (PBS, Sigma-Aldrich, Munich, Germany) were freshly prepared, stored at $+4^{\circ} \mathrm{C}$ and used within 2 months (see Supplementary Table S1 for details). Polymerization was initiated by addition of $1 / 1,000 \mathrm{~N}, \mathrm{~N}, \mathrm{~N}, \mathrm{~N}-$ tetramethylethylenediamine (TEMED) and 1/100 ammonium persulfate (APS) solution. Thirty-five microliter of this solution was used per cover slip. A square hydrophobic cover glass was placed on top in order to equally distribute the solution on the bottom glass. Gels were polymerized for $60 \mathrm{~min}$ in a saturated water atmosphere to avoid evaporation and were $\sim 70 \mu \mathrm{m}$ thick. Young's modulus $E$ of PAA gels was quantitatively controlled by measurements on a bulk rheometer (MCR-501, Anton Paar, Austria). To prevent toxicity to cells, non-polymerized residues were thoroughly washed away using PBS. Substrates were treated with the photo-activatable cross-linker Sulfo-SANPAH (Thermo Scientific, Waltham, Massachusetts, 22,589; $0.4 \mathrm{mM}$ in $50 \mathrm{mM}$ HEPES buffer at $\mathrm{pH} 8)$, exposed to UV light $(\lambda=365 \mathrm{~nm})$ for $10 \mathrm{~min}$ and then either coated with rat tail collagen I $(0.02$ $\mathrm{mg} / \mathrm{mL}$ for data presented in all main figures and $0.2 \mathrm{mg} / \mathrm{mL}$ for Supplementary Figure S3) (Corning, New York, New York, \#354236) or fibrinogen $(0.02 \mathrm{mg} / \mathrm{mL}$, from human plasma, Sigma, Munich, Germany \# F-3879) overnight at $4^{\circ} \mathrm{C}$ unless otherwise stated.

\section{Human Neutrophil Isolation}

All experiments with human neutrophils were approved by the Ethics Committee of the University Medical Center (UMG) Göttingen (protocol number: 29/1/17). Donors gave informed voluntary consent to the study. Neutrophils were isolated according to previously published standard protocols $(3,35)$ from healthy donors' venous blood supplemented with EDTA.

In short, fresh blood was collected with S-Monovettes KE $7.5 \mathrm{ml}$ (Sarstedt, Nümbrecht, Germany). Blood was gently layered in a 1:1 ratio on top of Histopaque 1119 (Sigma-Aldrich, Munich, Germany) and centrifuged at $1,100 \times g$ for $21 \mathrm{~min}$. Then, the transparent third and pink fourth layer containing the white blood cells were collected and mixed with HBSS (without $\mathrm{Ca}^{2+} / \mathrm{Mg}^{2+}$, Thermo Fisher Scientific, Waltham, Massachusetts). Cells were pelleted by centrifugation for $10 \mathrm{~min}$ at $400 \times g$. After discarding the supernatant, the pellet was resuspended in HBSS without $\mathrm{Ca}^{2+} / \mathrm{Mg}^{2+}$ and layered on top of a phosphate buffered percoll (GE Healthcare, Chicago, Illinois) gradient with the concentrations $85,80,75,70$, and $65 \%$ and centrifuged at $1,100 \times g$ for $22 \mathrm{~min}$. The accumulated neutrophils were received by collecting half of the $70 \%$, full $75 \%$ and half of the $80 \%$ layer and washed with HBSS. The remaining cell pellet was resuspended in $1 \mathrm{ml}$ HBSS. Cells were counted using Trypan 


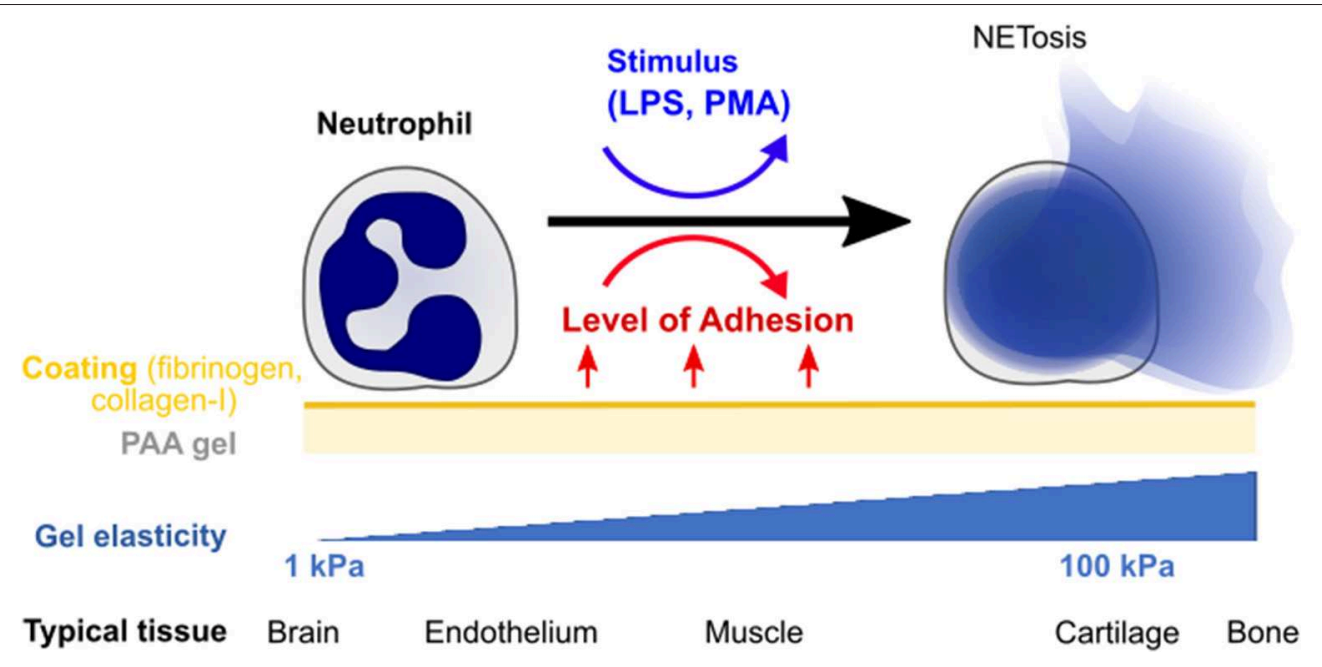

FIGURE 1 | Quantifying the impact of substrate elasticity, adhesion, and stimulation on NETosis. Human neutrophils are cultured on polyacrylamide (PAA) gels of different elasticity/stiffness and coating to control and vary adhesion. Cells are then stimulated with PMA or LPS to assess the impact of the different environmental factors on NETosis. The bottom row provides biological references for stiffnesses in different tissues.

blue solution (Sigma, Munich, Germany) and cell viability was assessed simultaneously. Viability was always $>98 \%$. The desired cell number was resuspended in Roswell Park Memorial Institute (RPMI) 1640 medium (Lonza, Basel, Schweiz) containing 0.5\% heat inactivated fetal calf serum (FCS; Biochrom GmbH, Berlin, Germany). Neutrophil purity was determined by a cytospin assay (Shanson, Cytospin 2 Centrifuge) followed by Diff Quick staining (Medion Diagnostics, Miami, USA). In all experiments neutrophil purity was $>95 \%$.

\section{Quantification of Neutrophil Extracellular Trap Formation}

Freshly isolated neutrophils were seeded $\left(0.5 \times 10^{6}\right.$ cells/well $)$ on PAA gels and collagen I- or fibrinogen-coated glass and incubated for $30 \mathrm{~min}$ at $37^{\circ} \mathrm{C}, 5 \% \mathrm{CO}_{2}$ in order to allow the cells to adhere. NETosis was either stimulated with $5 \mathrm{nM}$ Phorbol-12-myristate-13-acetate (PMA, Sigma-Aldrich, Munich, Germany) or with $75 \mu \mathrm{g} / \mathrm{mL}$ lipopolysaccharide (LPS) from Pseudomonas aeruginosa (serotype 10.22, strain: ATCC 27316, Sigma-Aldrich, Munich, Germany) and incubated for $3 \mathrm{~h}\left(37^{\circ} \mathrm{C}, 5 \% \mathrm{CO}_{2}\right)$. As vehicle control, neutrophils on 4 and $128 \mathrm{kPa}$ gels coated with collagen-I or fibrinogen were incubated with RPMI media for $3 \mathrm{~h}$ to control for spontaneous NETosis. NETosis was stopped with $2 \%$ paraformaldehyde (PFA, Roth, Karlsruhe, Germany) fixation overnight. The next day, the fixed neutrophil chromatin was stained using $1 \mu \mathrm{g} / \mathrm{ml}$ Hoechst 33342, trihydrochloride, trihydrate (Hoechst) (life technologies, Carlsbad, California). Cells were imaged with an Axiovert 200 microscope (16× magnification, Zeiss, Oberkochen, Germany) being set on the blue channel (Filter set49 DAPI shift free, 488049-9901-000, Zeiss, Oberkochen, Germany) connected to a CoolSNAP ES camera (Photometrics, Tucson, Arizona). Six images of different locations were taken per well. Phase contrast images for cell contact area measurements were taken for each setting and the cell contact areas were calculated by using ImageJ $4.46 \mathrm{r}$ for at least 50 cells per condition. NETosis was quantified in a standardized blinded fashion as percentage of condensed/lobulated nuclei vs. decondensed nuclei and strands of extracellularly localized chromatin, as described previously in the literature (36). For better clarity, we used "decondesed chromatin" when labeling axes.

\section{Immunofluorescence Staining}

Human neutrophils were isolated, seeded (200,000-500,000/well) in 24-well plates on glass coverslips and activated to undergo NET formation as described above. After fixation with 2\% PFA (Roth, Karlsruhe, Germany) over night, cells were permeabilized $0.1 \%$ TritonX (Merck, 165 Darmstadt, Germany) and incubated with a 5\%-FCS solution (Biochrom, Berlin, Germany) or the blocking solution (from TSA-kit, Perkin Elmer, Waltham, Massachusetts) to block unspecific antibody binding. Subsequently, cells were stained using monoclonal anti-human MPO (IgG, mouse) as primary antibody (Abcam, Cambridge, UK, ab25989, 1:500) and the polyclonal anti-mouse Alexa 555 (Life technologies, Carlsbad, California, IgG, goat, A21422, 1:2,000) or the antimouse Alexa488 secondary antibody (Cell Signaling Technology, Danvers, Massachusetts, IgG, goat, \#4408, 1:300) as secondary antibody. Neutrophil DNA was stained with $1 \mu \mathrm{g} / \mathrm{ml}$ Hoechst (Sigma-Aldrich, Munich, Germany) as described above. After the staining procedure, cells were stored protected from light at $4^{\circ} \mathrm{C}$. Representative fluorescence images were obtained with the olympus IX83 inverted confocal microscope (software: Olympus Fluoview Ver.4.2, Olympus, Tokio, Japan) and recorded $60 \times$ magnified (UPlanSApo 1.35 oil, Olympus, Tokio, Japan) or with the Axiovert 200 (Zeiss, Oberkochen, Germany, software: MicroManager 1.4.22) $40 \times$ magnified (Zeiss LD Achroplan $40 \times / 0.60$ Corr. Ph2, Zeiss, Oberkochen, Germany). 


\section{PI3K Inhibition}

Human neutrophils were treated with the PI3K inhibitor BAY 806946 (copanlisib) for $20 \mathrm{~min}$ on ice and then seeded on PAA gels $(4,128 \mathrm{kPa})$ and glass followed by a $30 \mathrm{~min}$ incubation $\left(37^{\circ} \mathrm{C}\right.$, $5 \% \mathrm{CO}_{2}$ ). Afterwards cells were stimulated, fixed, and imaged as described above.

\section{Neutrophil Extracellular Trap Formation on Passivated Glass Surfaces}

Human neutrophils (10,000 cells/well) were seeded on glass surfaces coated with $0.5 \mathrm{mg} / \mathrm{mL}$ Poly-L-lysine (PLL, Sigma, Munich, Germany) or Poly-L-lysine-grafted-polyethylenglycol (PLL-g-PEG (SuSoS Surface Technology, Dübendorf, Switzerland) and incubated for $30 \mathrm{~min}$ as described before (28). Uncoated glass surfaces were used as controls. Then cells were stimulated with $5 \mathrm{nM}$ PMA or $150 \mu \mathrm{g} / \mathrm{ml}$ LPS (to ensure maximum activation and, if possible, adhesion), fixed and stained as described above. For Reflection Interference Contrast Microscopy (RICM) a special objective was used (63× magnified EC Plan-Neofluar Ph3 objective/420481-9911000, 1.6× Optovar, Zeiss, Oberkochen, Germany) and also a RICM filter set (reflector module Pol ACR P\&Cfor HBO 100/ 424924-9901-000 and emission filter 416 LP, AHF-Nr.: F76-416/000000-1370-927, Zeiss, Oberkochen, Germany).

\section{Statistics and Data Analysis}

Statistics were performed with GraphPadPrism (Version 6.0, GraphPadSoftware Inc., San Diego, California). Significance was tested using standard one-way-ANOVA with Bonferroni's multiple comparisons test ( $\mathrm{ns}=$ not significant, ${ }^{*} p<0.05$, $\left.{ }^{* *} p<0.01,{ }^{* * *} p<0.001,{ }^{* * * *} p<0.0001\right)$, after testing for normal distribution, where applicable. $n$ numbers refer to the number of single cells analyzed, $\mathrm{N}$ numbers refer to the number of independent donors. Mean results and standard error of the mean (SEM) were calculated on the basis of the results obtained from the independent donors. Fluorescence images were processed with ImageJ.46r (National Institutes of Health, Bethesda, Maryland) and all cell counts obtained using the Plugin "Cell Counter."

\section{RESULTS}

\section{Substrate Elasticity Affects NETosis in a Stimulant-Dependent Manner}

To investigate the effect of substrate elasticity/stiffness on NETosis, freshly isolated human neutrophils were seeded on PAA gels coated with either collagen I or fibrinogen which are both known to interact with integrins on neutrophils $(30,37)$. The PAA substrate elasticity was varied within the physiological range $(1,2,4,8,16,20,30,64,128 \mathrm{kPa})$. The prepared gels cover a wide range of physiological elasticities for example that of brain tissue $(<1 \mathrm{kPa})$, muscle $(14-16 \mathrm{kPa})$, osteoids, pre-calcified bone (30 kPa) or cartilage (>100 kPa) (9). Importantly, PAA gels without coating do not provide a physiological surface and do not promote cell adhesion. This coating procedure leads to a uniform high density distribution of the proteins on the surface and was kept constant for different gels to ensure that possible differences in neutrophil behavior were not due to increased availability of surface interaction partners $(13,38)$.

To understand how substrate elasticity and the presence of certain integrin ligands affect NETosis, neutrophils were seeded and incubated for $30 \mathrm{~min}$ and then stimulated with either PMA or LPS for $3 \mathrm{~h}$ (Figure 2; Supplementary Figures S1, S2). As vehicle control, neutrophils on 4 or $128 \mathrm{kPa}$ PAA collagen-I- or fibrinogen-coated gels were incubated with RPMI media only and did not show significant NET formation (Supplementary Figures S1, S2). PMA is a well-known activator of protein kinase $\mathrm{C}(\mathrm{PKC})$ and frequently used to induce NETosis in vitro (39). LPS on the other hand, induces NETosis in a receptor-mediated fashion (40). PMA was applied at a very low concentrations $(5 \mathrm{nM})$ to avoid the strong stimulation at typical concentrations [100 nM (3)] that could blur the impact of substrate elasticity.

Formation of NETs was confirmed (Figures 2B,C on glass and Supplementary Figure $\mathbf{5} 5$ on gels) by co-staining of decondensed intracellular or released extracellular chromatin by Hoechst and MPO, according to commonly used protocols (41). As histone citrullination in PMA- induced NETosis is highly controversial, staining of citrullinated histones as a means of verifying NET formation was not carried out $(42,43)$.

NETosis was imaged and quantified on both surface coatings (collagen I and fibrinogen) for both ways of stimulation (PMA and LPS) (Figure 2). Representative images for 2, 16, $128 \mathrm{kPa}$ PAA gels and a glass control are shown in Figure 2. Importantly, NETs can be distinguished from apoptotic cells by their characteristic, decondensed chromatin. Counting decondensed nuclei and extracellular chromatin is a standard procedure to quantify NET-formation rates (36).

Images of all other conditions are shown in the Supplementary Figures S1, S2. PMA-stimulated NETosis was independent of stiffness. On collagen I-coated substrates, the NETosis rate was the same for stiffness values (Figure 3A). Similarly, on fibrinogen-coated substrates, PMA-induced NETosis (Figure 3C) did not vary with stiffness. However, on glass surfaces, NETosis was higher compared to that on substrates with defined stiffnesses of $8,16,20$, or $128 \mathrm{kPa}$, respectively. This observation might be explained by different effective concentrations of PMA due to diffusion into the gel or adsorption onto glass. We concluded that PMA-induced NETosis is not affected by substrate stiffness.

In contrast, NETosis was significantly affected by substrate elasticity under LPS stimulation on both collagen and fibrinogencoated surfaces (Figures 3B,D). On stiffer substrates $(E>20$ $\mathrm{kPa}$ ), NETosis was significantly increased. Interestingly, NET formation on collagen-I coated glass surfaces was comparably low after LPS stimulation, which correlated well with poor spreading of neutrophils on said glass surfaces (see below, Figure 4A).

\section{Correlation Between Neutrophil Adhesion and NETosis}

To further investigate the connection between NETosis, adhesion and substrate elasticity we assessed neutrophil adhesion on the 


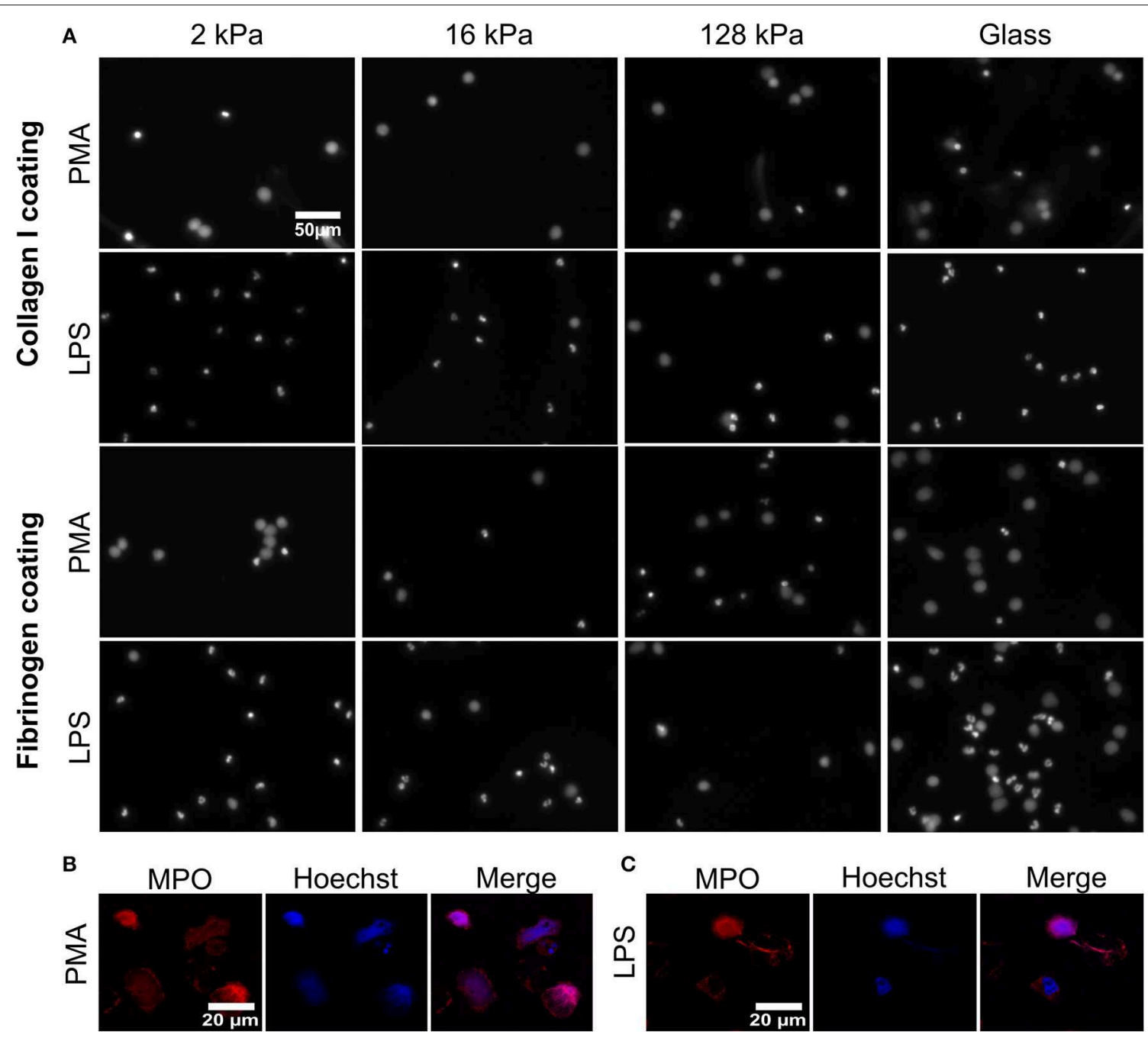

FIGURE 2 | Substrate elasticity affects NETosis in a stimulant-dependent manner. (A) Human neutrophils were seeded on PAA gels coated with collagen-I or fibrinogen. They were stimulated with either PMA $(5 \mathrm{nM})$ or LPS $(75 \mu \mathrm{g} / \mathrm{mL})$, as indicated, and incubated for $3 \mathrm{~h}$. The fluorescence images show the nuclei or extracellular chromatin (Hoechst DNA/chromatin stain) of fixed cells. The images reveal that NETosis depends on all three parameters (substrate elasticity, stimulant, surface coating). See quantification in Figure $\mathbf{3}$ and images of all conditions in Supplementary Figures S1, S2. (B,C) Neutrophils were seeded on glass coverslips and stimulated with PMA (B) or LPS (C) as described above. After fixation with PFA, cells were stained with anti-MPO antibody (red) and Hoechst against chromatin (blue) and imaged by confocal microscopy to verify NET production. Representative images of neutrophils are shown.

different substrates. Of note, fibrinogen is known to activate neutrophils and promote adhesion (37). However, as fibrinogen concentration was not varied, any changes in adhesion could be attributed to the changing stiffness of the substrate. Although it is known that upon stimulation with PMA or LPS neutrophils initially adhere strongly to the provided surfaces before starting NETosis (3), it remains controversial whether this initial adhesion is a necessary prerequisite and to which extent NETosis and adhesion are correlated with one another. During NETosis, the cells round up and the cytoskeleton is degraded. There have been contradictory studies on the role of integrin receptor engagement during NET formation $(33,34)$. The substrate stiffness most likely influences adhesion. Indeed, neutrophils have been reported to better adhere to stiff surfaces compared to soft surfaces $(17,18)$. Therefore, we next analyzed the spreading of human neutrophils on collagen I- and fibrinogencoated substrates and investigated the correlation between adhesion and NETosis. As a surrogate parameter for adhesion we determined the cell contact area (by phase contrast microscopy, representative images shown in Supplementary Figure S4). On both fibrinogen and collagen I-coated PAA gels the cell contact area/spreading area of neutrophils increased with increasing stiffnesses (Figures 4A,B), corresponding to the measured LPSinduced NETosis rates (Figures 3B,D). Next, a cross-correlation analysis for neutrophil spreading area vs. NETosis was carried out for LPS stimulation. LPS-induced NETosis correlated well with spreading area for both coatings (Figures 4C,D). Therefore, adhesion signaling appears to be highly relevant for LPS-induced 

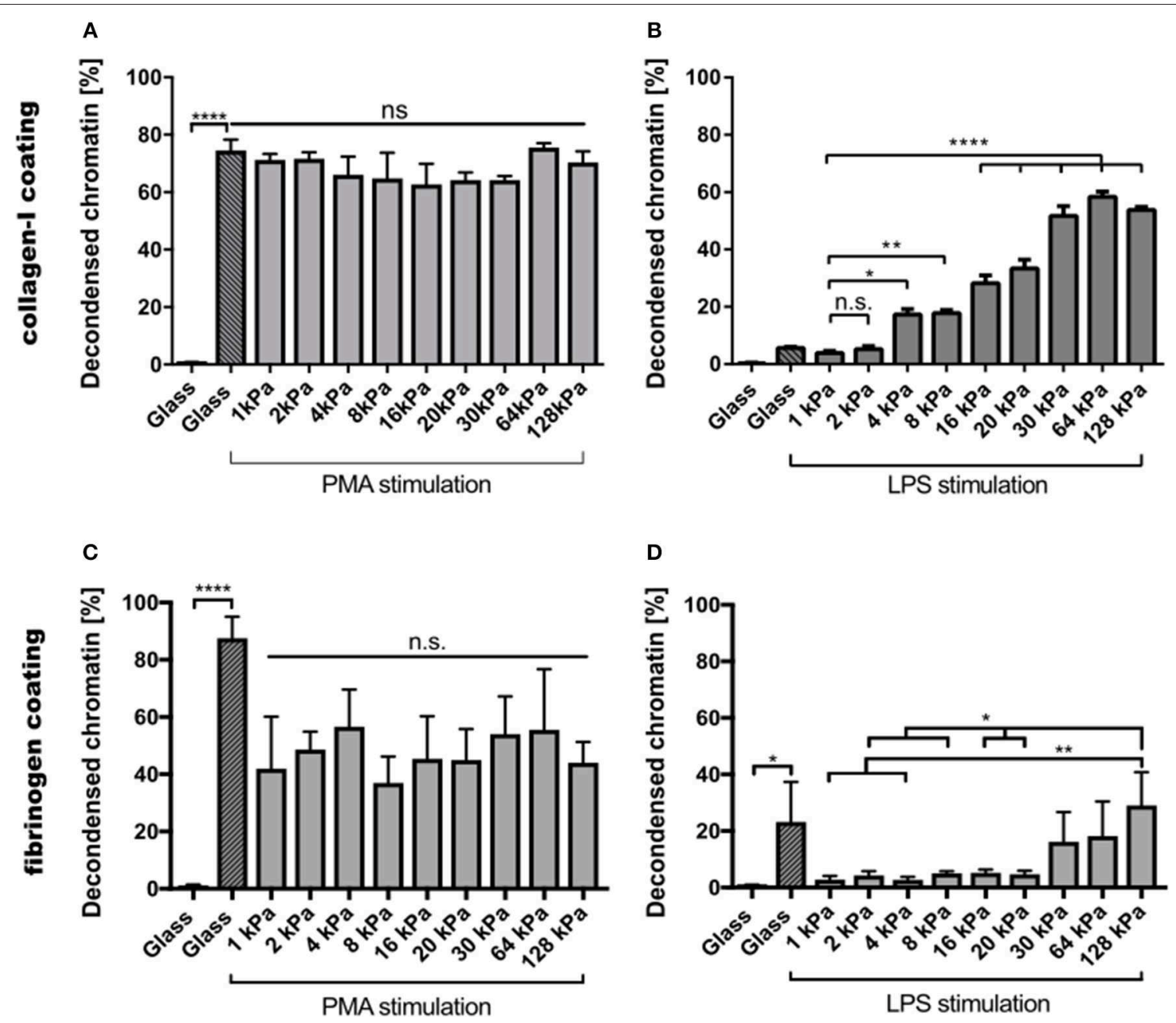

FIGURE 3 | NETosis depends on substrate elasticity, stimulant, and surface coating. Human neutrophils were seeded on PAA gels and glass surfaces coated with either collagen I (A,B) or fibrinogen (C,D), and stimulated with either PMA (5 nM) (A,C) or LPS $(75 \mu \mathrm{g} / \mathrm{mL})$ (B,D) and incubated for $3 \mathrm{~h}$. PMA-induced NETosis does not depend on substrate elasticity. LPS-induced NETosis increases significantly for both coatings above a stiffness threshold of $>20 \mathrm{kPa}$. $n>500$ cells for each condition. $N=3$ donors. Statistics: one-way ANOVA (Bonferroni's multiple comparisons test; ${ }^{\star} p<0.05 ;{ }^{* \star} p<0.01$; ${ }^{\star \star \star \star} p<0.0001$; ns: not significant). Mean \pm SEM of independent donors.

NETosis. Differences in adhesion due to different stiffnesses directly translate into different NETosis rates.

In contrast, as expected from the results in Figure 3, PMA-induced NETosis did not correlate with the spreading area (Figure 4E). Interestingly, when the gels were coated with a 10 -fold higher $(0.2 \mathrm{mg} / \mathrm{ml})$ collagen I concentration, cells did not perform LPS-induced NETosis at all (Supplementary Figure S3). We hypothesized that there is an optimum density for NET formation on collagen I, in accordance with previously published results that show an optimum ligandreceptor ratio for different biological functions including cell adhesion or spreading $(24,28,44,45)$. Similarly, the cells did not adhere well and did not spread above the area expected from a fully settled neutrophil (Supplementary Figure S3). These observations additionally corroborate our hypothesis that conditions that affect the adhesive phenotype change
NETosis rates, which suggests that adhesion and NETosis are interconnected.

\section{PI3K Inhibition Abrogates}

\section{Stiffness-Dependent Variations in NETosis}

PI3K activity is important for neutrophil mechanosensing and enables these cells to distinguish between substrates of different stiffnesses (18). To corroborate that the observed effects on LPSinduced NETosis could be attributed to variations in substrate stiffness, we treated neutrophils with a potent PI3K inhibitor, BAY 80-6946 (copanlisib), and analyzed adhesion and NETosis. Fibrinogen-coated PAA gels of 4 and $128 \mathrm{kPa}$, respectively, were chosen as representative conditions for adhesion and NETosis quantification as they had revealed significant differences in the experiments presented above. Adhesion was impaired when neutrophils on stiff surfaces (128 kPa and glass) were pre-treated 


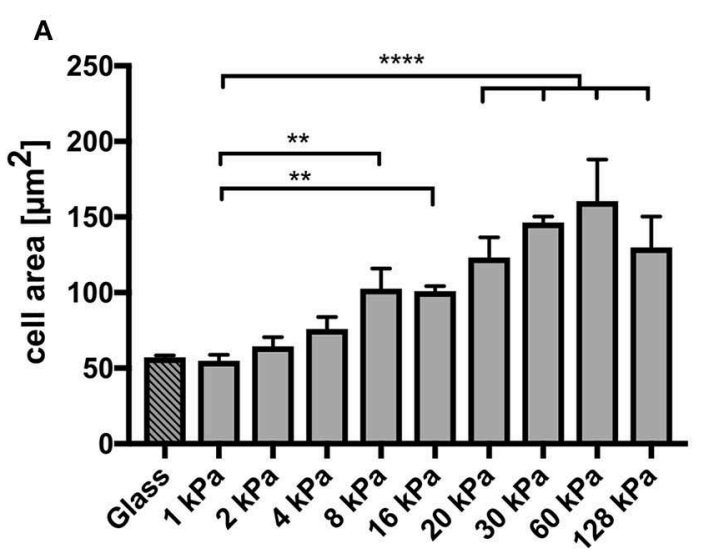

C

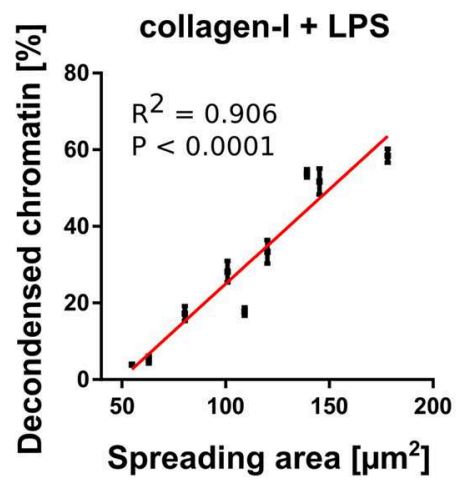

D

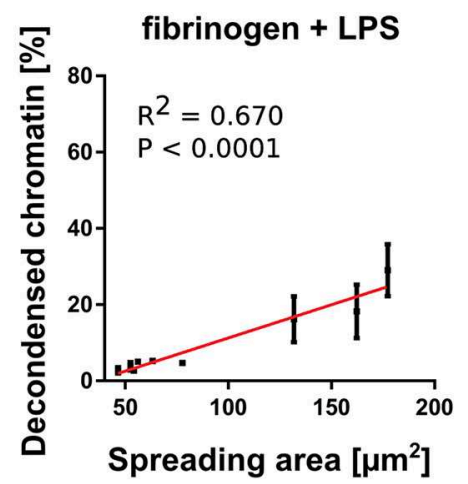

B

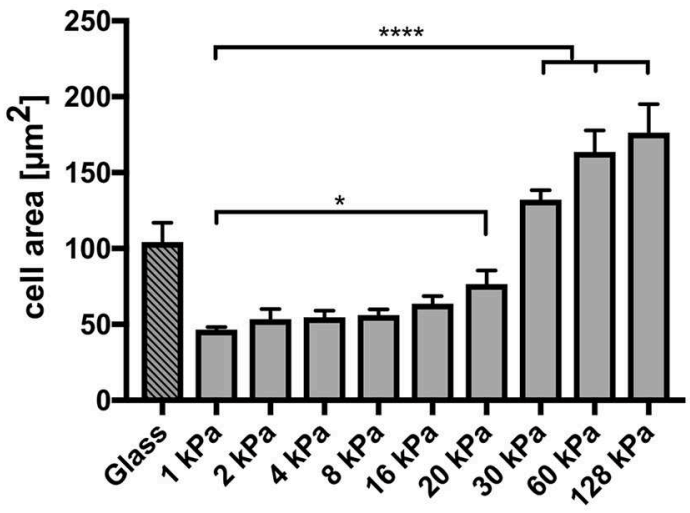

FIGURE 4 | NETosis correlates with cell spreading area. (A) Cell spreading area increases with increasing stiffness on collagen I-coated PAA gels. (B) Cell spreading area increases with increasing stiffness on fibrinogen-coated PAA gels. LPS-stimulated NETosis correlates with spreading area on PAA gels of different stiffness coated with collagen (C) and fibrinogen (D). (E) PMA stimulation does not depend on spreading area. The red lines indicate linear fits. $R^{2}$ and $P$-values (indicating likelihood of slope being non-zero) are shown in the graph for each condition (C). $n>500$ cells for each condition. $N=3$ donors. Mean \pm SEM of independent donors. Statistics: one-way ANOVA (Bonferroni's multiple comparisons test. ${ }^{*} p<0.05 ;{ }^{* \star} p<0.01$; ${ }^{* * * *} p<0.0001$; ns: not significant).

with the inhibitor (Figures $\mathbf{5 A}, \mathbf{B}$ ). PI3K is a known signaling intermediate for PMA-induced NETosis, which is why moderate effects on PMA-induced NETosis were to be expected (40). To investigate the connection between adhesion and NETosis, we treated cells with PMA or LPS under PI3K inhibition. This treatment partially impaired PMA-induced (Figure 5C), and completely abrogated LPS-induced NETosis (Figure 5D).

To further prove the importance of adhesion for NETosis, we coated glass surfaces with poly-L-lysine (PLL) and polyL-lysine-grafted-polyethylene glycol (PLL-g-PEG). PEG functionalization/passivation is well-established to prevent unspecific adsorption of proteins and adhesion of cells (28). This environment therefore does not provide any adhesive cues and can be used to test how adhesion affects NETosis. First, cells were seeded on glass, PLL-coated, and PLL-g-PEG-coated surfaces and imaged by using reflection interference contrast microscopy (RICM). Dark regions in RICM images indicate close proximity between the cell and the substrate (i.e., adhesion), while bright regions indicate non-adhesive sitting of the cell on the substrate. Cells adhered to both glass and PLL-coated surfaces, with images suggesting a slightly stronger adhesion on glass (Figures 6A,B). However, they did not adhere to PLL-g-PEG coated surfaces as expected (Figure 6C). Cells were stimulated on these coatings and NETosis was found to be independent from adhesion under PMA stimulation as indicated previously (3) (Figure 6D). On the other hand, LPS-induced NETosis was completely inhibited on PLL-g-PEG, even with very high concentrations of LPS $(150 \mu \mathrm{g} / \mathrm{ml})$ (Figure 6E).

\section{DISCUSSION}

Neutrophils are the most abundant leukocytes in humans. Being the first responders to inflammation, neutrophils infiltrate different kinds of tissue and subsequently face different mechanical and chemical environments. Substrate elasticity has been shown to influence important cellular functions such as adhesion, differentiation and migration of many different types of cells including neutrophils $(10,11,13,38,46)$. Therefore, we investigated for the first time how NETosis is affected by 
A

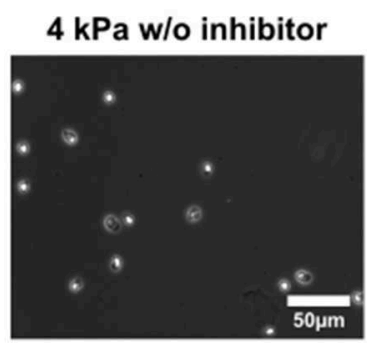

$128 \mathrm{kPa}$ w/o inhibitor
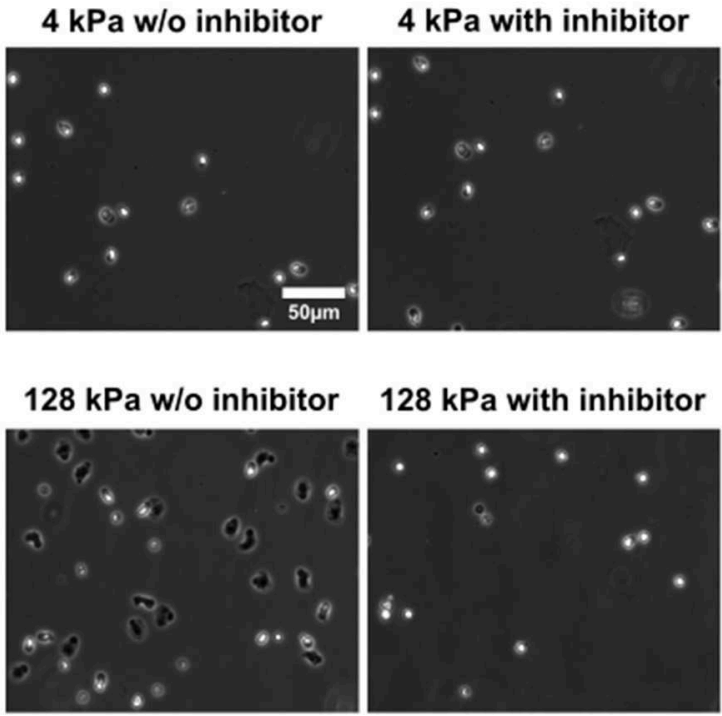

C

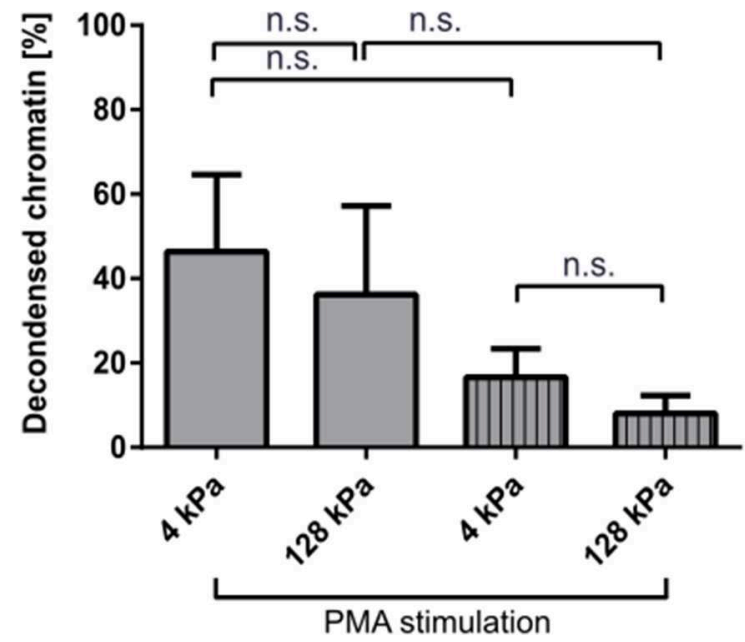

B

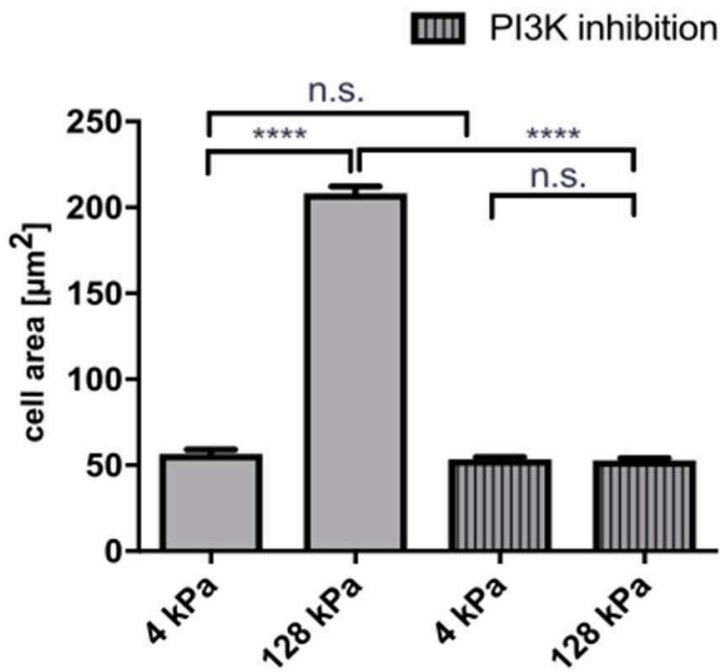

D

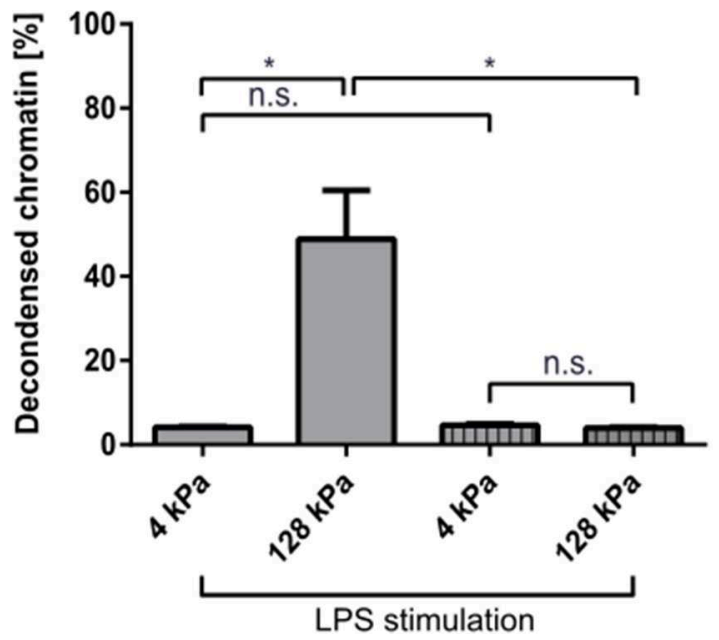

FIGURE 5 | PI3K inhibition impairs neutrophil adhesion and affects NETosis in a stimulant-dependent manner. Human neutrophils were seeded on fibrinogen-coated PAA gels (4 and $128 \mathrm{kPa}$ ) and glass (serving as a control) and the spreading area was quantified with and without PI3K inhibition after 30 min (A,B). Representative phase contrast images are shown in (A). PI3K inhibition leveled out the elasticity-dependent differences in cell contact area. Then NETosis was induced by PMA (5 nM) (C) or LPS $(75 \mu \mathrm{g} / \mathrm{mL})$ (D) and quantified after $3 \mathrm{~h}$. LPS-induced NETosis is completely abolished. In contrast, PMA-induced NETosis is only partially reduced by PI3K inhibition. $n>500$ cells per condition. $N=3$ donors. Mean \pm SEM of independent donors. Statistics: one-way ANOVA (Bonferroni's multiple comparisons test. ${ }^{\star} p<0.05 ;{ }^{* \star * \star} p<0.0001$; ns: not significant).

substrate elasticity in general and more specifically by substrate elasticity-modulated adhesion. Our results clearly indicate that LPS-mediated NETosis depends on the cells' adhesion and correlates with the adhesion/contact area, which itself correlates with stiffness.

NETosis is initiated and transmitted via diverse pathways, highly depending on the respective stimulus. PI3K is part of the intermediate signaling in PMA-induced and plateletinduced NETosis (40). PI3K also plays an important role in neutrophil mechanosensing and has been shown to be required for neutrophils to sense substrates of higher stiffness (18). In order to investigate the role of mechanosensing on neutrophil spreading as well as on NETosis, we tested a highly selective PI3K inhibitor (BAY 80-6946, copanlisib). Indeed, PI3K inhibition impaired spreading of neutrophils and subsequently LPS-induced NETosis. It also inhibited PMA-induced NETosis to a certain degree, which is understandable as PI3-kinase plays a role in PMA-mediated activation of neutrophils. However, 

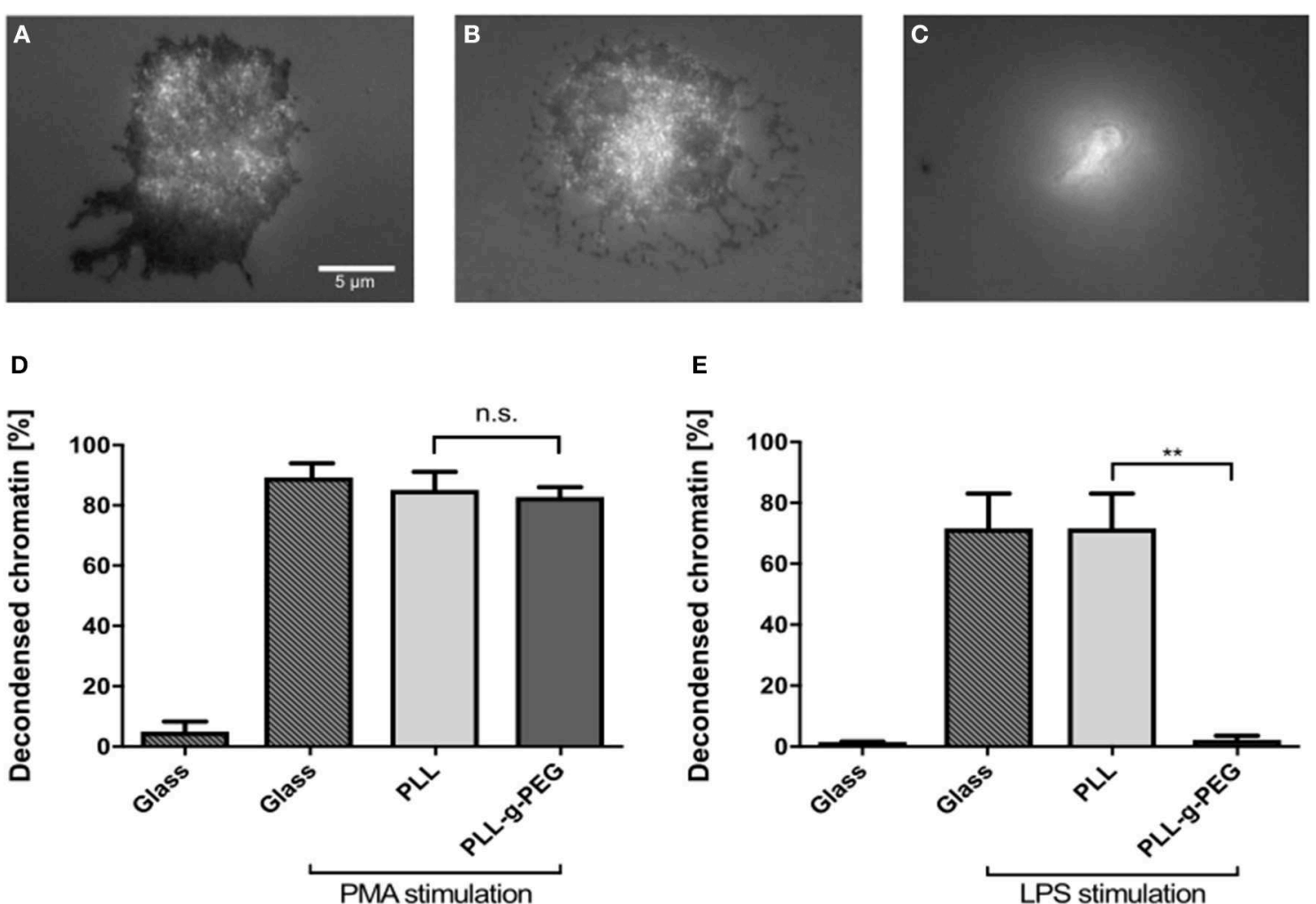

FIGURE 6 | LPS-induced NETosis but not PMA-induced NETosis requires adhesion. RICM images of fixed cells cultured on glass (A), PLL (B), and PLL-g-PEG (C) coated glass surfaces. Compared to glass and PLL surfaces, neutrophils cultured on PLL-g-PEG show no adhesion (C). NETosis on these different substrates was quantified for PMA (5 nM) (D), and LPS (150 $\mathrm{g} / \mathrm{ml})$ stimulation (E). PMA-induced NETosis also took place on the passivated non-adhesive PLL-g-PEG surfaces, while LPS-induced NETosis did not occur. $n>500$ cells for each condition. $N=3$ donors. Statistics: One-way ANOVA (Bonferroni's multiple comparisons test ${ }^{* \star} p<0.01$; ns: not significant). Mean \pm SEM of independent donors.

it did not abrogate NET formation completely as it did after LPS-stimulation.

Another factor affecting cell adhesion is the available surface concentration of integrin ligands $(25,44)$. By using nanotechnology approaches it is possible to control the exact distance and overall density of integrin ligands such as RGD and the Mac-1 ligand GPB1 $\alpha$ or even link them to advanced near infrared fluorescent nanomaterials $(28,47,48)$. For neutrophils, adhesion maturation and cellular functions such as spreading and migration depend on ligand density (28). Here, we showed that on collagen, LPS-induced NETosis not only depends on substrate elasticity but also on the amount of available surface cues. On a substrate that provided a very high density of surface cues (collagen I) cells did not adhere very well and consequently NETosis did not take place. For PMA, NETosis was once again independent of surface cues, in this case density of surface receptors. Indeed, PMA-induced NETosis does not appear to require adhesion at all (Figure 6). These differences between the stimulants can be explained, at least to some extent, by the different receptor and signaling pathways involved in PMA- and LPS-stimulated NETosis. PMA acts intracellularly and directly activates protein kinase $\mathrm{C}(\mathrm{PKC})$. It also triggers subsequent production of reactive oxygen species (ROS), which then interact with MEK, ERK, PI3K, mTOR, MPO, and NE (2, 49, 50). LPS also activates PKC in neutrophils, primarily through binding to toll-like receptor 4 (TLR4) (51). Our results show that there is an active connection between adhesion and stiffness signaling and LPS-triggered NETosis, putatively by a connection between integrin and TLR4 signaling, which remains to be explored in depth in the context of NETosis. This hypothesis is also corroborated by the complete abrogation of LPS-induced NETosis following phosphatidylinositol 3-kinase (PI3K) blocking. PMA-induced NETosis is also decreased after PI3K inhibition, thus indicating a role of PI3K in PMA-signaling. However, NETosis still took place, albeit at a lower level. It is important to stress that the medium conditions of NETosis assays affect absolute NETosis rates and might shift certain thresholds as previously shown (41).

Our results might bear considerable medical and pharmacological implications as neutrophils and other cells of the immune system are continuously confronted with surfaces of different stiffness/elasticity. One may speculate that reducing neutrophil responses on and in tissues with low substrate elasticity such as the brain or most importantly the 
blood itself may serve to keep aberrant immune responses in check. On the other hand, alterations of tissue stiffness as seen in arteriosclerotic vessels, tumor tissue, or organ fibrosis might lead to an increase of inflammatory NETosis-related processes $(52,53)$. Indeed, NET-induced inflammation has been implicated in fibrotic organ changes and is likely to trigger tissue remodeling and lead to even more tissue stiffness, causing a pro-inflammatory, self-sustaining vicious circle (54). Our results may also be of importance to the explanation of how NETosis can be triggered by implant materials (55), which tend to have a higher stiffness than "natural" tissues within the body. An improved understanding of the environmental factors that affect NETosis could therefore lead to novel therapeutic approaches for diseases that coincide with alterations of tissue elasticity such as arteriosclerosis, lung, or liver fibrosis or cancer. This understanding will also be of great importance not only for unifying and understanding of NETosis experiments in general, but also for the design of future implant and exogenous materials, which are designed to remain in the body for a certain time, such as catheters.

\section{CONCLUSION}

In summary, we show how NETosis rates are affected by different levels of substrate elasticity and adhesion. Neutrophil adhesion increases with substrate stiffness, which leads to higher LPSinduced NETosis rates on stiff substrates. In contrast, PMAinduced NETosis does not require any adhesion at all.

\section{DATA AVAILABILITY STATEMENT}

The datasets generated during and/or analyzed during the current study are available from the corresponding author on reasonable request.

\section{REFERENCES}

1. Brinkmann V, Reichard U, Goosmann C, Fauler B, Uhlemann Y, Weiss DS, et al. Neutrophil extracellular traps kill bacteria brinkmann science 2004.pdf. Science. (2004) 303:1532-5. doi: 10.1126/science.1092385

2. Fuchs TA, Abed U, Goosmann C, Hurwitz R, Schulze I, Wahn V, et al. Novel cell death program leads to neutrophil extracellular traps. J Cell Biol. (2007) 176:231-41. doi: 10.1083/jcb.200606027

3. Neubert E, Meyer D, Rocca F, Günay G, Kwaczala-Tessmann A, Grandke J, et al. Chromatin swelling drives neutrophil extracellular trap release. Nat Commun. (2018) 9:3767. doi: 10.1038/s41467-018-06263-5

4. Nauseef WM, Borregaard N. Neutrophils at work. Nat Immunol. (2014) 15:602-11. doi: 10.1038/ni.2921

5. Barnado A, Crofford LJ, Oates JC. At the bedside: neutrophil extracellular traps (NETs) as targets for biomarkers and therapies in autoimmune diseases. J Leukoc Biol. (2016) 99:265-78. doi: 10.1189/jlb.5BT0615-234R

6. Demers M, Wagner DD. Neutrophil extracellular traps. Oncoimmunology. (2013) 2:e22946. doi: 10.4161/onci.22946

\section{ETHICS STATEMENT}

The studies involving human participants were reviewed and approved by Ethik-Kommission der Universitätsmedizin Göttingen. The patients/participants provided their written informed consent to participate in this study.

\section{AUTHOR CONTRIBUTIONS}

SK and LE designed the study. AG, GG, DM, EN, JB, and LE performed NETosis experiments. LE, AG, MS, and SK analyzed data. GK and FR prepared and characterized gels. LE, AG, GG, and SK wrote the manuscript with inputs from all authors.

\section{FUNDING}

This project was supported by the state of Lower Saxony (life@nano) and the German Research Foundation (DFG grant KR 4242/4-1 and ER 723/2-1). FR acknowledges funding through the DFG within the SFB 937 project A13.

\section{ACKNOWLEDGMENTS}

This manuscript has been released as Pre-Print at BioRxiv (56). We thank Andreas Janshoff and Claudia Steinem for fruitful discussions and support. We are grateful for fruitful discussions about active matter with members of the collaborative research center SFB 937 funded by the DFG. We thank Meike Shaffrinsky for technical support.

\section{SUPPLEMENTARY MATERIAL}

The Supplementary Material for this article can be found online at: https://www.frontiersin.org/articles/10.3389/fimmu. 2019.02320/full\#supplementary-material

7. Döring Y, Soehnlein O, Weber C. Neutrophil extracellular traps in atherosclerosis and atherothrombosis. Circ Res. (2017) 120:736-43. doi: 10.1161/CIRCRESAHA.116.309692

8. Erpenbeck L, Chowdhury CS, Zsengeller ZK, Gallant M, Burke SD, et al. PAD4 deficiency decreases inflammation and susceptibility to pregnancy loss in a mouse model. Biol Reprod. (2016) 95:132. doi: 10.1095/biolreprod.116.1 40293

9. Rehfeldt F, Engler AJ, Eckhardt A, Ahmed F, Discher DE. Cell responses to the mechanochemical microenvironment-Implications for regenerative medicine and drug delivery. Adv Drug Deliv Rev. (2007) 59:1329-39. doi: 10.1016/j.addr.2007.08.007

10. Discher DE. Tissue cells feel and respon to the stiffness of their substrate. Science. (2005) 310:1139-43. doi: 10.1126/science.1116995

11. Engler AJ, Sen S, Sweeney HL, Discher DE. Matrix elasticity directs stem cell lineage specification. Cell. (2006) 126:677-89. doi: 10.1016/j.cell.2006.06.044

12. Lee-Thedieck C, Rauch N, Fiammengo R, Klein G, Spatz JP. Impact of substrate elasticity on human hematopoietic stem and progenitor cell adhesion and motility. J Cell Sci. (2012) 125:3765-75. doi: 10.1242/jcs.095596 
13. Zemel A, Rehfeldt F, Brown AEX, Discher DE, Safran SA. Optimal matrix rigidity for stress-fibre polarization in stem cells. Nat Phys. (2010) 6:468-73. doi: $10.1038 /$ nphys 1613

14. Adlerz KM, Aranda-Espinoza H, Hayenga HN. Substrate elasticity regulates the behavior of human monocyte-derived macrophages. Eur Biophys J. (2016) 45:301-9. doi: 10.1007/s00249-015-1096-8

15. Mennens SFB, Bolomini-Vittori M, Weiden J, Joosten B, Cambi A, Van Den Dries K. Substrate stiffness influences phenotype and function of human antigen-presenting dendritic cells. Sci Rep. (2017) 7:1-14. doi: 10.1038/s41598-017-17787-Z

16. Okamoto T, Takagi Y, Kawamoto E, Park EJ, Usuda H, Wada K, et al. Reduced substrate stiffness promotes M2-like macrophage activation and enhances peroxisome proliferator-activated receptor $\gamma$ expression. Exp Cell Res. (2018) 367:264-73. doi: 10.1016/j.yexcr.2018. 04.005

17. Jannat RA, Robbins GP, Ricart BG, Dembo M, Hammer DA. Neutrophil adhesion and chemotaxis depend on substrate mechanics. J Phys Condens Matter. (2010) 22:194117. doi: 10.1088/0953-8984/22/19/1 94117

18. Oakes PW, Patel DC, Morin NA, Zitterbart DP, Fabry B, Reichner JS, et al. Neutrophil morphology and migration are affected by substrate elasticity. Blood. (2009) 114:1387-95. doi: 10.1182/blood-2008-11-191445

19. Stroka KM, Aranda-Espinoza H. Neutrophils display biphasic relationship between migration and substrate stiffness. Cell Motil Cytoskeleton. (2009) 66:328-41. doi: 10.1002/cm.20363

20. Stroka KM, Aranda-Espinoza H. Endothelial cell substrate stiffness influences neutrophil transmigration via myosin light chain kinase-dependent cell contraction. Blood. (2011) 118:1632-40. doi: 10.1182/blood-2010-11-3 21125

21. Matsumoto T, Abe H, Ohashi T, Kato Y, Sato M. Local elastic modulus of atherosclerotic lesions of rabbit thoracic aortas measured by pipette aspiration method. Physiol Meas. (2002) 23:635-48. doi: 10.1088/0967-3334/23/ $4 / 304$

22. Georges PC, Hui J-J, Gombos Z, McCormick ME, Wang AY, Uemura $\mathrm{M}$, et al. Increased stiffness of the rat liver precedes matrix deposition: implications for fibrosis. AJP Gastrointest Liver Physiol. (2007) 293:G1147-54. doi: 10.1152/ajpgi.00032.2007

23. Denis M, Gregory A, Bayat M, Fazzio RT, Whaley DH, Ghosh K, et al. Correlating tumor stiffness with immunohistochemical subtypes of breast cancers: prognostic value of comb-push ultrasound shear elastography for differentiating luminal subtypes. PLoS ONE. (2016) 11:e0165003. doi: 10.1371/journal.pone.0165003

24. Amschler K, Kossmann E, Erpenbeck L, Kruss S, Schill T, Schön M, et al. Nanoscale tuning of VCAM-1 determines VLA-4-dependent melanoma cell plasticity on RGD motifs. Mol Cancer Res. (2018) 16:528-42. doi: 10.1158/1541-7786.MCR-17-0272

25. Kruss S, Wolfram T, Martin R, Neubauer S, Kessler H, Spatz JP. Stimulation of cell adhesion at nanostructured teflon interfaces. Adv Mater. (2010) 22:5499506. doi: 10.1002/adma.201003055

26. Kruss S, Srot V, van Aken PA, Spatz JP. Au-Ag hybrid nanoparticle patterns of tunable size and density on glass and polymeric supports. Langmuir. (2012) 28:1562-8. doi: 10.1021/la204395d

27. Haston WS, Shields JM, Wilkinson PC. Lymphocyte locomotion and attachment on two-dimensional surfaces and in three-dimensional matrices. $J$ Cell Biol. (1982) 92:747-52. doi: 10.1083/jcb.92.3.747

28. Kruss S, Erpenbeck L, Amschler K, Mundinger TATA, Boehm H, Helms H-JH-J, et al. Adhesion maturation of neutrophils on nanoscopically presented platelet glycoprotein Ib $\alpha$. ACS Nano. (2013) 7:9984-96. doi: $10.1021 / \mathrm{nn} 403923 \mathrm{~h}$

29. Lämmermann T, Bader BL, Monkley SJ, Worbs T, Wedlich-Söldner R, Hirsch $\mathrm{K}$, et al. Rapid leukocyte migration by integrin-independent flowing and squeezing. Nature. (2008) 453:51-5. doi: 10.1007/978-3-642-42051-1_40

30. Monboisse JC, Garnotel R, Randoux A, Dufer Borel JJP. Adhesion of human neutrophils to and activation by type-I collagen involving a 2integrin. J Leukoc Biol. (1991) 50:373-80. doi: 10.1002/jlb.50.4.373

31. Yipp BG, Kubes P, Dc W, Yipp BG, Kubes P. NETosis : how vital is it? Bloo. (2013) 122:2784-94. doi: 10.1182/blood-2013-04-457671
32. Raftery MJ, Lalwani P, Krautkrämer E, Peters T, Scharffetter-Kochanek K, Krüger R, et al. $\beta 2$ integrin mediates hantavirus-induced release of neutrophil extracellular traps. J Exp Med. (2014) 211:1485-97. doi: 10.1084/jem.201 31092

33. Mohanty T, Sjögren J, Kahn F, Abu-Humaidan AHA, Fisker N, Assing K, et al. A novel mechanism for NETosis provides antimicrobial defense at the oral mucosa. Blood. (2015) 126:2128-38. doi: 10.1182/blood-2015-04-641142

34. Rossaint J, Herter JM, Van Aken H, Napirei M, Doring Y, Weber $\mathrm{C}$, et al. Synchronized integrin engagement and chemokine activation is crucial in neutrophil extracellular trap-mediated sterile inflammation. Blood. (2014) 123:2573-84. doi: 10.1182/blood-2013-07-5 16484

35. Brinkmann V, Laube B, Abu Abed U, Goosmann C, Zychlinsky A. Neutrophil extracellular traps: how to generate and visualize them. J Vis Exp. (2010) 24:1724. doi: 10.3791/1724

36. Wong SL, Demers M, Martinod K, Gallant M, Wang Y, Goldfine $\mathrm{AB}$, et al. Diabetes primes neutrophils to undergo NETosis, which impairs wound healing. Nat Med. (2015) 21:815-9. doi: 10.1038/nm. 3887

37. Rubel C, Fernandez GC, Dran G, Bompadre MB, Isturiz MA, Palermo MS. Fibrinogen promotes neutrophil activation and delays apoptosis. J Immunol. (2001) 166:2002-10. doi: 10.4049/jimmunol.166. 3.2002

38. Kaliman S, Jayachandran C, Rehfeldt F, Smith A-S. Novel growth regime of MDCK II model tissues on soft substrates. Biophys J. (2014) 106:L25-8. doi: 10.1016/j.bpj.2013.12.056

39. Hoppenbrouwers T, Autar ASA, Sultan AR, Abraham TE, Van Cappellen WA, Houtsmuller AB, et al. In vitro induction of NETosis: comprehensive live imaging comparison and systematic review. PLoS ONE. (2017) 12:1-29. doi: 10.1371/journal.pone.0176472

40. Papayannopoulos V. Neutrophil extracellular traps in immunity and disease. Nat Rev Immunol. (2018) 18:134-47. doi: 10.1038/nri.2017.105

41. Neubert E, Senger-Sander SN, Manzke VS, Busse J, Polo E, Scheidmann $\mathrm{SEF}$, et al. Serum and serum albumin inhibit in vitro formation of Neutrophil Extracellular Traps (NETs). Front Immunol. (2019) 10:12. doi: 10.3389/FIMMU.2019.00012

42. de Bont CM, Koopman WJH, Boelens WC, Pruijn GJM. Stimulusdependent chromatin dynamics, citrullination, calcium signalling and ROS production during NET formation. Biochim Biophys Acta Mol Cell Res. (2018) 1865:1621-9. doi: 10.1016/J.BBAMCR.2018. 08.014

43. Kenny EF, Herzig A, Krüger R, Muth A, Mondal S, Thompson PR, et al. Diverse stimuli engage different neutrophil extracellular trap pathways. Elife. (2017) 6:e24437. doi: 10.7554/eLife.24437

44. Kruss S, Erpenbeck L, Schön MP, Spatz JP. Circular, nanostructured and biofunctionalized hydrogel microchannels for dynamic cell adhesion studies. Lab Chip. (2012) 12:3285. doi: 10.1039/c2lc40611j

45. Amschler K, Erpenbeck L, Kruss S, Schön MP. Nanoscale integrin ligand patterns determine melanoma cell behavior. ACS Nano. (2014) 8:9113-25. doi: $10.1021 / \mathrm{nn} 502690 \mathrm{~b}$

46. Rehfeldt F, Brown AEX, Raab M, Cai S, Zajac AL, Zemel A, et al. Hyaluronic acid matrices show matrix stiffness in $2 \mathrm{D}$ and $3 \mathrm{D}$ dictates cytoskeletal order and myosin-II phosphorylation within stem cells. Integr Biol. (2012) 4:422. doi: $10.1039 / \mathrm{c} 2 \mathrm{ib} 00150 \mathrm{k}$

47. Mann FA, Horlebein J, Meyer NF, Meyer D, Thomas F, Kruss S. Carbon nanotubes encapsulated in coiled-coil peptide barrels. Chem A Eur J. (2018) 24:12241-5. doi: 10.1002/chem.201800993

48. Polo E, Nitka TT, Neubert E, Erpenbeck L, Vuković L, Kruss S. Control of integrin affinity by confining RGD peptides on fluorescent carbon nanotubes. ACS Appl Mater Interfaces. (2018) 10:17693-703. doi: 10.1021/acsami.8b04373

49. Hakkim A, Fuchs TA, Martinez NE, Hess S, Prinz H, Zychlinsky $A$, et al. Activation of the Raf-MEK-ERK pathway is required for neutrophil extracellular trap formation. Nat Chem Biol. (2011) 7:75-7. doi: $10.1038 /$ nchembio.496

50. Papayannopoulos V, Metzler KD, Hakkim A, Zychlinsky A. Neutrophil elastase and myeloperoxidase regulate the formation of neutrophil 
extracellular traps. J Cell Biol. (2010) 191:677-91. doi: 10.1083/jcb.2010 06052

51. Clark SR, Ma AC, Tavener SA, McDonald B, Goodarzi Z, Kelly MM, et al. Platelet TLR4 activates neutrophil extracellular traps to ensnare bacteria in septic blood. Nat Med. (2007) 13:463-9. doi: 10.1038/nm1565

52. Butcher DT, Alliston T, Weaver VM. A tense situation: forcing tumour progression. Nat Rev Cancer. (2009) 9:108-22. doi: 10.1038/nrc2544

53. Wells RG. Tissue mechanics and fibrosis. Biochim Biophys Acta Mol Basis Dis. (2013) 1832:884-90. doi: 10.1016/j.bbadis.2013.02.007

54. Martinod K, Witsch T, Erpenbeck L, Savchenko A, Hayashi H, Cherpokova D, et al. Peptidylarginine deiminase 4 promotes age-related organ fibrosis. J Exp Med. (2017) 214:439-58. doi: 10.1084/jem.20160530

55. Jhunjhunwala S, Aresta-DaSilva S, Tang K, Alvarez D, Webber MJ, Tang BC, et al. Neutrophil responses to sterile implant materials. PLoS ONE. (2015) 10:1-16. doi: 10.1371/journal.pone.0137550
56. Erpenbeck L, Gruhn AL, Kudryasheva G, Guenay G, Meyer D, Neubert E, et al. Effect of adhesion and substrate elasticity on neutrophil extracellular trap formation. bioRxiv [Preprint]. (2019). doi: 10.1101/508366

Conflict of Interest: The authors declare that the research was conducted in the absence of any commercial or financial relationships that could be construed as a potential conflict of interest.

Copyright (C) 2019 Erpenbeck, Gruhn, Kudryasheva, Günay, Meyer, Busse, Neubert, Schön, Rehfeldt and Kruss. This is an open-access article distributed under the terms of the Creative Commons Attribution License (CC BY). The use, distribution or reproduction in other forums is permitted, provided the original author(s) and the copyright owner(s) are credited and that the original publication in this journal is cited, in accordance with accepted academic practice. No use, distribution or reproduction is permitted which does not comply with these terms. 